\title{
Hydraulic model optimization of a multi-product pipeline
}

\author{
Liang Yongtu ${ }^{1 *}$ Li Ming ${ }^{2}$ and Li Jiangfei ${ }^{1}$ \\ ${ }^{1}$ School of Petroleum Engineering, China University of Petroleum (Beijing), Beijing 102249, China \\ ${ }^{2}$ Sinopec Engineering Corporation, Beijing 100101, China \\ (C) China University of Petroleum (Beijing) and Springer-Verlag Berlin Heidelberg 2012
}

\begin{abstract}
An optimization model is established for a multi-product pipeline which has a known delivery demand and operation plan for each off-take station. The aim of this optimization model is to minimize the total pumping operation cost, considering not only factors including the energy equilibrium constraint, the maximum and minimum suction and discharge pressures constraints of pump stations, and pressure constraint at special elevation points, but also the regional differences in electricity prices along the pipeline. The dynamic programming method is applied to solve the model and to find the optimal pump configuration.
\end{abstract}

Key words: Multi-product pipeline, pump sets, regional electricity price, optimization, dynamic programming

\section{Introduction}

Products of different physical properties, such as diesel and various gasolines, transported in the same pipeline, result in the pressure characteristics along the pipeline being more complicated than that of a single product pipeline (Gong and Yu, 1992; Rejowski and Pinto, 2004; Tang et al, 2011). The greatest feature of a multi-product pipeline is batch transportation. Variations in the pump station characteristics and pipeline characteristics caused by batch movement of different products in the pipeline, and delivery/injection operations along the pipeline result in changes of the configuration of pump sets of the whole pipeline. Different configurations of pump sets impose diverse pipeline operation costs. Many studies have been performed on power cost optimization, especially concerning optimizing configurations of pump sets to achieve minimum pumping power cost while ensuring operation safety and satisfying the delivery requirements. The optimal pump configuration in previous studies has been mainly determined by considering constraint conditions such as maximum and minimum suction and discharge pressures, pressures of high-elevation points, and speed range of the control motor, with a constant electricity price assumption (Liang, 2004). In China, a multi-product pipeline usually crosses many areas. Because of the long distances, regional electricity prices may be considerably different owing to various electricity generating costs. Therefore, the assumption of a constant electricity price is not suitable for Chinese domestic conditions. So it is necessary to establish an optimization model for pipeline operations in which the regional differences in electricity prices are taken into account.

The pipeline operators press for effective off-line simulation software for optimizing multi-product pipeline operations. SCICLOPS from Britain, PACOS developed by the Pichler company in German and SPS by the STONER company in America are widely used in the pipeline simulation field, but no standardized software for multiproduct pipeline simulation developed in China. On the basis of optimization theory, a mathematical model for optimizing operations of multi-product pipeline has been developed using dynamic programming. The software STROBER for multiproduct pipeline operation simulation has been developed.

\section{Target function of the model}

The optimal configuration of pumps and the minimum throttling volume in pump stations can be calculated in order to minimize total electricity cost and to ensure the most efficient and economic operation of a multi-product pipeline. A multi-product pipeline system can be modeled using an energy conservation equation. This equation must meet the following constraints: The flow rate through the initial pump station should be as stable as possible over a long period. The inlet and outlet pressures of pump stations and pressures of some special points should be within the preset limits and the delivery task should be completed during the prescribed time (Liang et al, 2004; Rejowski and Pinto, 2003; Méndez and Cerdá, 2003). The target function of this model is given by: 


$$
\left.\min F=\sum_{j=1}^{J N} \sum_{i=1}^{I N} \sum_{u=1}^{U N_{j}} \frac{W_{i, u}^{j} S_{i} \rho_{i}^{j} g Q_{i}^{j}\left(A_{i, u} \frac{\left(n_{i, u}^{j}\right)^{2}}{\left(n_{i, u}^{r}\right)^{2}}+B_{i, u}\left(Q_{i}^{j}\right)^{7 / 4}\right)}{3.6 \times 10^{6} \times\left(a_{i, u} \frac{\left(n_{i, u}^{j}\right)^{2}}{\left(n_{i, u}^{r}\right)^{2}}\left(Q_{i}^{j}\right)^{2}+b_{i, u} \frac{n_{i, u}^{j}}{n_{i, u}^{r}} Q_{i}^{j}+c_{i, u}\right.}\right)^{t_{j}}
$$

\section{Model constraints}

\subsection{Energy use along the pipeline}

$$
P_{\mathrm{s}}^{j}+\sum_{i=1}^{I N} \sum_{u=1}^{U N_{i}} H_{i, u}^{j} \rho_{i}^{j} g=F_{\mathrm{h}}^{j}+V_{\text {cut }}^{j}+P_{\mathrm{e}}^{j}+L_{1}+F^{j}
$$

The above equation represents energy use of the multiproduct pipeline system at the $j$ th time step. In a multi-product pipeline system, the pump sets are connected in series. The equation will be modified correspondingly when the pump sets are connected in parallel.

\subsection{Pressure constraints at special points}

These special points refer to some points at low and high elevations along the pipeline. Super-high pressure at the lowest elevation point and vaporization at the highest elevation point may occur. To avoid these extremes, the pressure constraints for these special points are as below:

$$
P_{\text {spe_min }}^{g} \leq P_{g, \text { spe }}^{j} \leq P_{\text {spe_max }}^{g}
$$

\subsection{Discharge pressure constraint}

Considering the pressure bearing capacity of the pipeline and associated downstream equipment, the discharge pressure should meet the following constraint at the $j$ th time step:

$$
P_{i, \mathrm{~d}}^{j} \leq P_{i, \mathrm{~d} \max }^{j}
$$

In this equation, the preset constraint of the discharge pressure should be given at each time step under different conditions.

\subsection{Suction pressure constraint}

In order to consider the allowable bearing pressure of the pump station equipment and the allowable suction pressure, the suction pressure should meet the following constraint at the $j$ th time step:

$$
P_{i, \mathrm{smin}}^{j} \leq P_{i, \mathrm{~s}}^{j} \leq P_{i, \mathrm{smax}}^{j}
$$

In the equation, the preset constraint of the maximum and minimum suction pressures should be given at each time step.

\subsection{Delivery task constraint}

The end of a transport cycle can be described as:

$$
V=\sum_{j=1}^{J N} Q_{1}^{j} \Delta t_{j}
$$

\subsection{Maximum delivery capacity constraint}

Ignoring the temperature variation of fluids, the maximum delivery capacity of a single-fluid pipeline is constant over a long period. However, for a long-distance pipeline having several pump stations, the suction and discharge pressures of each pump station, together with the pressures at special points, vary with transport time due to fluid flow and variation of the product batches in the pipeline. In addition, the pressure variation in the pipeline, caused by start-up and shut-down of the off-take stations along the pipeline, results in a change in the maximum delivery capacity of the pipeline (Cafaro and Cerdá, 2004; Hui and Gupta, 2000; Hui et al, 2000).

When multiple products or batches are transported in a pipeline, the maximum delivery capacity of the pipeline should be constantly determined to maximize pipeline utilization. The maximum delivery capacity can be calculated with methods proposed by other researchers (Prasad and Maravelias, 2008; Cafaro and Cerdá, 2008; 2010). The input flow rate of the initial station must meet the constraint as indicated below:

$$
Q_{1}^{j} \leq Q_{\max }^{j}
$$

\section{Model analysis}

The aim of this model is to minimize the electricity cost of pump stations. Therefore, the optimal operation scheme is that one which can most effectively minimize the electricity cost in the operation cycle.

To obtain the optimization result of pump sets, work can be divided into several procedures. Each stage is likely to have different decisions. Therefore, this optimization is a multi-stage decision problem. The most commonly used method to solve multi-stage decision problems is dynamic programming theory. The main idea of dynamic programming theory is the "optimization principle", namely, for a multistage decision process - whatever their front process strategies are - the optimal strategy depends only on the current state. Based on this theory, many researchers have proposed a series of solutions.

For each interval between stations, the problem of pipeline pressure distribution can be divided into several phases. Assuming that the problem is divided into $n$ phases and $x_{p}$ represents the start of the $p$ th phase, $x_{p}(1 \leq p \leq n)$ represents the state variables of each state. Variable sets of each state are described as $x_{p}=\left\{x_{p}^{1}, x_{p}^{2}, \cdots, x_{p}^{n}\right\}$. The decision variable of the $p$ th state is the effective pressure head provided by the $p$ th pump station, namely $u_{p}=\sum_{u=1}^{U N_{p}}\left(H_{p, u}^{j} \times W_{p, u}^{j}\right)$. The allowable decision set can be described as $U_{p}\left(x_{p}\right)$. The state transfer equation can be described as $x_{p+1}=x_{p}+u_{p}$.

Constraint conditions: State variables $x_{\mathrm{p}}$ in the model meet a series of constraints: the maximum and minimum pressure constraints at special points along the pipeline and the maximum and minimum suction/discharge pressure constraints of each pump station: 


$$
\begin{aligned}
& A_{p}^{j} \leq x_{p} \leq B_{p}^{j} \\
& A_{p}^{j}=\max \left(A 1_{p}^{j}, A 2_{p}^{j}\right) \\
& B_{p}^{j}=\min \left(B 1_{p}^{j}, B 2_{p}^{j}, B 3_{p}^{j}\right)
\end{aligned}
$$

Minimum suction pressure constraint:

$$
\mathrm{Al}_{p}^{j}=P_{p+1, \mathrm{smin}}^{j}-P_{\mathrm{s}}^{j}+F_{p+1}^{j}+F_{\mathrm{h}, p+1}^{j}+L_{1, p+1}+V_{\text {cut }, p+1}^{j}
$$

Pressure constraint of higher points of elevation:

$$
\mathrm{A} 2_{p}^{j}=P_{\text {spe } \_ \text {min }, p+1}-P_{\mathrm{s}}^{j}+\overline{F_{p+1}^{j}}+\overline{F_{\mathrm{h}, p+1}^{j}}+L_{1, p+1}+V_{\text {cut }, p+1}^{j}
$$

Maximum suction pressure constraint:

$$
B 1_{p}^{j}=P_{p+1, \mathrm{~s} \max }^{j}-P_{\mathrm{s}}^{j}+F_{p+1}^{j}+F_{\mathrm{h}, p+1}^{j}+L_{1, p+1}+V_{\mathrm{cut}, p+1}^{j}
$$

Maximum discharge pressure constraint:

$$
B 2_{p}^{j}=P_{p, \mathrm{~d} \max }^{j}-P_{\mathrm{s}}^{j}+F_{p}^{j}+F_{\mathrm{h}, p}^{j}+L_{\mathrm{l}, p+1}+V_{\mathrm{cut}, p+1}^{j}
$$

Pressure constraint of lower points of elevation:

$$
B 3_{p}^{j}=P_{\text {spe_max }, p+1}-P_{\mathrm{s}}^{j}+\overline{F_{p+1}^{j}}+\overline{F_{\mathrm{h}, p+1}^{j}}+L_{1, p+1}+V_{\mathrm{cut}, p+1}^{j}
$$

\section{Model solution}

The optimization of a multi-product pipeline is different from that of a crude oil pipeline or a natural gas pipeline. Hydraulic conditions along the pipeline change owing to fluid flow and variation of product batches, and frequent start/ stop operations of each distribution station. Therefore, pump optimization is required for the entire simulation process.

In order to reduce the memory size required for the calculation and to increase the computational speed, a primary hydraulic calculation is needed to extract pump set combinations that do not work. The primary hydraulic calculation is based on actual information, mainly about physical characteristics of the piping system, physical properties of oil, characteristics of pump units, flow rate of the initial station, and some basic requirements for pipeline operations. An effective state set (Wu, 1992) can be obtained through an integrated method of state propagation, function propagation, and removing invalid state sets. Therefore, the optimal solution to the model can then be obtained.

\section{Case study}

The Southwestern Multi-product Pipeline transports $0^{\#}$ diesel, 90 $0^{\#}$ gasoline, and $93^{\#}$ gasoline in batches, with a designed transportation capacity of $1,000 \times 10^{4} \mathrm{t} / \mathrm{a}$. The pipeline has two off-take stations, two pump stations, ten offtake and pump stations, and one terminal station.

Table 1 lists the operation data of the Southwestern Pipeline in March, 2008. The optimal result for the pump set is obtained after optimization using the dynamic programming model mentioned above. Then, electricity costs of the pipeline operation with and without considering regional electricity price differences are calculated separately, as shown in Tables 2 and 3, respectively.

Table 1 Product demands at each off-take station

\begin{tabular}{cccccc}
\hline \multirow{2}{*}{ Station } & \multicolumn{5}{c}{ Product demand, $\mathrm{m}^{3}$} \\
\cline { 2 - 6 } Yulin & 0 & 0 & 1326.3 & 11834.3 & 1326.3 \\
Guigang & 0 & 0 & 2652.5 & 11834.3 & 1061.0 \\
Nanning & 0 & 0 & 5305.0 & 23668.6 & 1326.3 \\
Litang & 0 & 0 & 2652.5 & 9467.5 & 265.3 \\
Liuzhouku & 0 & 591.7 & 10610.1 & 21301.8 & 0 \\
Hechi & 0 & 1775.2 & 2652.5 & 11834.3 & 0 \\
Duyun & 0 & 5917.2 & 5305.0 & 11834.3 & 0 \\
Guiyang & 0 & 18934.9 & 7957.6 & 9467.5 & 0 \\
Anshun & 0 & 13017.8 & 3978.8 & 1183.4 & 0 \\
Qinglong & 0 & 11834.3 & 3978.8 & 5917.2 & 0 \\
Panxian & 0 & 11834.3 & 3978.8 & 9467.5 & 0 \\
Qujing & 0 & 30769.2 & 2652.5 & 18934.9 & 0 \\
Yangtianchong & 132.6 & 18934.9 & 1326.3 & 23668.6 & 0 \\
\hline
\end{tabular}

Notes: Batch 1, Batch 3 and Batch 5 are 93\# gasoline; Batch 2 and Batch 4 are $0 \#$ diesel.

Table 2 indicates that when considering regional differences in electricity prices, the total electricity cost is minimized by adjusting the electricity consumption of each station, namely by enabling the stations with high electricity price to use less electricity while the stations with low electricity price use more electricity.

In order to further analyze the impact of regional differences in electricity prices, the optimal electricity cost considering the impact of electricity price is compared with the electricity cost of the actual operation and results are shown in Table 3.

As shown in Table 3, both the electricity consumption and the operation cost of the optimal pump set considering regional differences of electricity prices are less than actual operation cost. It saves $54.51 \times 10^{4} \mathrm{CNY}$ and the economic benefit is obvious. Therefore, the optimal result considering regional difference of electricity price can be regarded as a reference for practical pipeline operation.

\section{Conclusions}

1) In this paper, a mathematical model is established to optimize the operation of a multi-product pipeline system, which can provide a guide for pipeline operators.

2) Based on the actual operation data of the Southwestern Multi-Product Pipeline, a mathematical model is developed to calculate the operation cost. After analyzing and contrasting the optimized results with the actual operation cost, it is 
Table 2 Electricity cost considering and without considering the regional differences in electricity prices

\begin{tabular}{|c|c|c|c|c|c|}
\hline \multirow{2}{*}{ Station } & \multicolumn{2}{|c|}{ Electricity consumption, $\mathrm{kWh}$} & \multirow{2}{*}{$\begin{array}{c}\text { Electricity price } \\
\mathrm{CNY} / \mathrm{kWh}\end{array}$} & \multicolumn{2}{|c|}{ Electricity cost, ten thousand $\mathrm{CNY}$} \\
\hline & Considering & Without considering & & Considering & Without considering \\
\hline Maoming & 1474244.0 & 1513976.0 & 0.67 & 98.28 & 100.94 \\
\hline Yulin & 1419399.0 & 1418691.0 & 0.47 & 66.68 & 66.65 \\
\hline Litang & 723518.30 & 740710.40 & 0.59 & 42.49 & 43.50 \\
\hline Liuzhou & 747250.30 & 767409.30 & 0.59 & 44.33 & 45.53 \\
\hline Hechi & 539721.50 & 802225.90 & 0.59 & 32.02 & 47.60 \\
\hline Luqiao & 850857.60 & 806564.10 & 0.44 & 37.17 & 35.23 \\
\hline Xiasi & 639808.20 & 638591.10 & 0.38 & 24.40 & 24.36 \\
\hline Duyun & 453503.30 & 526151.60 & 0.40 & 18.20 & 21.12 \\
\hline Guiyang & 388963.50 & 388116.50 & 0.40 & 15.61 & 15.58 \\
\hline Anshun & 404890.00 & 405617.10 & 0.40 & 16.25 & 16.28 \\
\hline Qinglong & 535675.20 & 534036.40 & 0.39 & 21.06 & 21.00 \\
\hline Panxian & 607271.10 & 576529.10 & 0.42 & 25.58 & 24.28 \\
\hline Yangtianchong & 88506.34 & 97944.88 & 0.48 & 4.29 & 4.75 \\
\hline Total & - & - & - & 446.38 & 466.81 \\
\hline
\end{tabular}

Table 3 A comparison of electricity costs between using regional price differences and actual flat-price cost

\begin{tabular}{|c|c|c|c|c|c|c|}
\hline \multirow[b]{2}{*}{ Station } & \multicolumn{3}{|c|}{ Actual data } & \multicolumn{3}{|c|}{ Electricity cost considering price differences } \\
\hline & $\begin{array}{l}\text { Electricity consumption } \\
\qquad \mathrm{kWh}\end{array}$ & $\begin{array}{l}\text { Electricity price } \\
\mathrm{CNY} / \mathrm{kWh}\end{array}$ & $\begin{array}{c}\text { Electricity cost } \\
10^{4} \mathrm{CNY}\end{array}$ & $\begin{array}{l}\text { Energy consumption } \\
\mathrm{kWh}\end{array}$ & $\begin{array}{l}\text { Electricity price } \\
\mathrm{CNY} / \mathrm{kWh}\end{array}$ & $\begin{array}{c}\text { Electricity cost } \\
10^{4} \mathrm{CNY}\end{array}$ \\
\hline Maoming & 1459496.00 & 0.67 & 97.30 & 1474244.00 & 0.67 & 98.28 \\
\hline Yulin & 1524400.00 & 0.47 & 71.62 & 1419399.00 & 0.47 & 66.68 \\
\hline Litang & 884000.00 & 0.59 & 51.92 & 723518.30 & 0.59 & 42.49 \\
\hline Liuzhou & 972940.00 & 0.59 & 57.72 & 747250.30 & 0.59 & 44.33 \\
\hline Hechi & 778740.00 & 0.59 & 46.20 & 539721.50 & 0.59 & 32.02 \\
\hline Luqiao & 1051200.00 & 0.44 & 45.92 & 850857.60 & 0.44 & 37.17 \\
\hline Xiasi (Xinshi Line) & 21000.00 & 0.38 & 0.80 & \multirow{2}{*}{639808.20} & \multirow{2}{*}{0.38} & \multirow{2}{*}{24.40} \\
\hline Xiasi (Xiashi Line) & 456750.00 & 0.38 & 17.42 & & & \\
\hline Duyun & 617940.00 & 0.40 & 24.80 & 453503.30 & 0.40 & 18.20 \\
\hline \multirow{2}{*}{ Guiyang } & 416139.00 & 0.40 & 16.70 & \multirow{2}{*}{388963.50} & \multirow{2}{*}{0.40} & \multirow{2}{*}{15.61} \\
\hline & 1113.00 & 0.65 & 0.07 & & & \\
\hline Anshun (Huashi Line) & 297800.00 & 0.40 & 11.95 & \multirow{2}{*}{404890.00} & \multirow{2}{*}{0.40} & \multirow{2}{*}{16.25} \\
\hline Anshun (Shuangshi Line) & 156000.00 & 0.40 & 6.26 & & & \\
\hline Qinglong (Qingsha Station) & 589400.00 & 0.39 & 23.18 & \multirow{2}{*}{535675.20} & \multirow{2}{*}{0.39} & \multirow{2}{*}{21.06} \\
\hline Qinglong (Qingda Line) & 73780.00 & 0.39 & 2.90 & & & \\
\hline \multirow{2}{*}{ Panxian } & 567450.00 & 0.42 & 23.90 & \multirow{2}{*}{607271.10} & \multirow{2}{*}{0.42} & \multirow{2}{*}{25.58} \\
\hline & 17550.00 & 0.68 & 1.19 & & & \\
\hline Yangtianchong & 140120.00 & 0.48 & 6.79 & 88506.34 & 0.48 & 4.29 \\
\hline Total & 9525818.00 & & 506.65 & 8873608.00 & & 446.38 \\
\hline
\end{tabular}


concluded that the optimal operation cost considering regional differences in electricity prices is significantly better than that of the actual cost.

3) Considering regional difference of electricity price along the pipeline is the core of this paper and it is the important innovation. However, this paper does not cover sensitivity analysis or stability of the model solution. They will be discussed in detail in the future work.

\section{Nomenclature}

$a_{i u}, b_{i u}, c_{i u} \quad$ Characteristic coefficients of the efficiency

$A_{i, u}, B_{i, u} \quad$ Constants determined by the characteristics of

$F^{j} \quad$ The total friction loss of the pipeline at the $j$ th

$F_{\mathrm{h}}^{j} \quad$ The total pressure loss due to elevation variation

$F_{p+1}^{j} \quad$ Friction loss along the pipeline between the initial

$\overline{F_{p+1}^{j}}$

$F_{\mathrm{h}, p+1}^{j} \quad$ Pressure loss due to elevation variation between time step, $\mathrm{Pa}$

$\overline{F_{\mathrm{h}, p+1}^{j}}$

$g$

$H_{i, u}^{j}$

$J N$

$L_{1}$

$L_{1, p+1}$

$n_{i, u}^{\mathrm{r}}$

$n_{i, u}^{j}$

$P_{\mathrm{e}}^{j}$

$P_{g \text {,spe }}^{j}$

$P_{i, \mathrm{~d}}^{j}$

$P_{i, \mathrm{~s}}^{j}$

$P_{i, \mathrm{~d} \max }^{j}$ performance curve of the $u$ th pump in the $i$ th station, dimensionless the $u$ th pump in the $i$ th station and pump unit combinations, dimensionless time step, $\mathrm{Pa}$ at the $j$ th time step, $\mathrm{Pa}$ station and the $(p+1)$ th station at the $j$ th time step, $\mathrm{Pa}$ Friction loss along the pipeline between the initial station and the high- or low-elevation points before the $(p+1)$ th station at the $j$ th time step, $\mathrm{Pa}$ the initial station and the $(p+1)$ th station at the $j$ th

$P_{i, \mathrm{smin}}^{j}$

$P_{i, \mathrm{~s} \max }^{j}$

$P_{p+1, \mathrm{smin}}^{j}$

$P_{p+1, \mathrm{smax}}^{j}$

$P_{p, \mathrm{~d} \max }^{j}$

$P_{\mathrm{s}}^{j}$

$P_{\text {spe_min }}^{g}$

$P_{\text {spe } \max }^{g}$

$P_{\text {spe } \min , p+1}$

$P_{\text {spe_max }, p+1}$

$Q_{i}^{j}$

$Q_{\max }^{j}$

$Q_{1}^{j}$

$S_{i}$

$\Delta t_{j}$

$U N_{i}$

V

Pressure loss due to elevation variation between the initial station and the high- or low-elevation points before the $(p+1)$ th station at the $j$ th time step, $\mathrm{Pa}$

Acceleration of gravity, $\mathrm{m}^{3} / \mathrm{s}$

Pressure head provided by the $u$ th pump unit in the $i$ th station at the $j$ th time step, $\mathrm{m}$

The number of time steps in a transportation cycle

The total local pressure loss of all the stations within the piping system, $\mathrm{Pa}$

The total friction loss within stations before the $(p+1)$ th station, $\mathrm{Pa}$

Rated speed of the $u$ th pump unit in the $i$ th station, r/min;

Speed of the $u$ th pump set in the $i$ th station at the $j$ th time step, $\mathrm{r} / \mathrm{min}$

Terminal pressure of pipeline at the $j$ th time step, $\mathrm{Pa}$

The pressure of special point $g$ at the $j$ th time step, $\mathrm{Pa}$

Discharge pressure of the $i$ th station at the $j$ th time step, $\mathrm{Pa}$

Suction pressure of the $i$ th station at the $j$ th time step, $\mathrm{Pa}$

Preset discharge pressure of the $i$ th pump station at the $j$ th time step, $\mathrm{Pa}$
Preset minimum suction pressure of the $i$ th station at the $j$ th time step, $\mathrm{Pa}$

Preset maximum suction pressure of the $i$ th station at the $j$ th time step, $\mathrm{Pa}$

Preset minimum suction pressure of the $(p+1)$ th station at the $j$ th time step, $\mathrm{Pa}$

Preset maximum suction pressure of the $(p+1)$ th station at the $j$ th time step, $\mathrm{Pa}$

Preset maximum discharge pressure of the $p$ th station at the $j$ th time step, $\mathrm{Pa}$

Pressure after the feed pump in the initial station at the $j$ th time step, $\mathrm{Pa}$

Preset minimum pressure of the gth special point, $\mathrm{Pa}$

Preset maximum pressure of the gth special point, $\mathrm{Pa}$

Preset minimum pressure of the high-elevation points before the $(p+1)$ th station, $\mathrm{Pa}$

Preset maximum pressure of the low-elevation point before the $(p+1)$ th station, $\mathrm{Pa}$

Flow rate of the $i$ th station at the $j$ th time step, $\mathrm{m}^{3} / \mathrm{s}$

Maximum transportation capacity at the $j$ th time step, $\mathrm{m}^{3} / \mathrm{s}$

Flow rate at the first station at the $j$ th time step, $\mathrm{m}^{3} / \mathrm{s}$

Electricity price in the $i$ th station, $\mathrm{CNY} / \mathrm{kWh}$

Step length of the $j$ th time step, $\mathrm{s}$

The number of pump sets in the $i$ th pump station

The total volume of fluids transporting in a pipeline in a transportation cycle, $\mathrm{m}^{3}$

$V_{\text {cut }}^{j} \quad$ The total throttle loss of the pipeline at the $j$ th time step, $\mathrm{Pa}$

$V_{\text {cut }, p+1}^{j} \quad$ The total throttling loss within stations before the $(p+1)$ th station, $\mathrm{Pa}$

$W_{i, u}^{j} \quad$ Operation state of the $u$ th pump unit in the $i$ th station at the $j$ th time step

$\rho_{i}^{j} \quad$ Product density at the $i$ th station at the $j$ th time step, $\mathrm{kg} / \mathrm{m}^{3}$

\section{Superscripts and subscripts}

\section{d Discharge pressure}

dmax Preset maximum discharge pressure

in Suction pressure

smax Preset maximum suction pressure

smin Preset minimum suction pressure

$g \quad$ Special point number

$i, p \quad$ Station number, and for the initial station, $i=1, p=1$

$j \quad$ Time step number

$u \quad$ Pump sets number

\section{References}

Cafaro D C and Cerdá J. Optimal scheduling of multiproduct pipeline systems using a non-discrete MILP formulation. Computers \& Chemical Engineering. 2004. 28(10): 2053-2068

Cafaro D C and Cerdá J. Dynamic scheduling of multiproduct pipelines with multiple delivery due dates. Computers \& Chemical Engineering. 2008. 32(4-5): 728-753 
Cafaro D C and Cerdá J. Operational scheduling of refined products pipeline networks with simultaneous batch injections. Computers \& Chemical Engineering. 2010. 34(10): 1687-1704

Gong $\mathrm{J}$ and Yu D. Simulated calculation of hydraulic characteristics for batching operation. Oil \& Gas Storage and Transportation. 1992. 11(2): 1-4 (in Chinese)

Hui C W and Gupta A. A novel MILP formulation for short-term scheduling of multistage multi-product batch plants. Computers \& Chemical Engineering. 2000. 24(2-7): 1611-1617

Hui C W, Gupta A and van der Meulen H A J. A novel MILP formulation for short-term scheduling of multi-stage multi-product batch plants with sequence-dependent constraints. Computers \& Chemical Engineering. 2000. 24(12): 2705-2717

Liang Y T, Gong J, Kang Z L. Research on operation optimization of multi-product pipeline. Proceeding of the ASME International Pipeline Conference, 4-8 October 2004, Calgary, Alberta, Canada. 2055-2060

Méndez C A and Cerdá J. Dynamic scheduling in multiproduct batch plants. Computers \& Chemical Engineering. 2003. 27(8-9): 12471259

Prasad P and Maravelias C T. Batch selection, assignment and sequencing in multi-stage multi-product processes. Computers \& Chemical Engineering. 2008. 32(6): 1106-1119

Rejowski Jr. R and Pinto J M. Scheduling of a multiproduct pipeline system. Computers \& Chemical Engineering. 2003. 27 (8-9): 12291246

Rejowski Jr. R and Pinto J M. Efficient MILP formulations and valid cuts for multiproduct pipeline scheduling. Computers \& Chemical Engineering. 2004. 28(8): 1511-1528

Tang Z C, Lu Z Z, Li D W, et al. Optimal design of the positions of the hoops for a hydraulic pipelines system. Nuclear Engineering and Design. 2011. 241(12): 4840-4855

Wu C C. Introduction of HOPOPT software pack for the optimization of thermal oil pipeline operation. Oil \& Gas Storage and Transportation. 1992. 1(2): 5-10 (in Chinese)

(Edited by Sun Yanhua) 